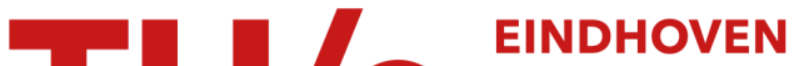 UNIVERSITY OF TECHNOLOGY
}

\section{Acoustic modes in a duct with slowly varying impedance and non-uniform mean flow and temperature}

\section{Citation for published version (APA):}

Oppeneer, M., Lazeroms, W. M. J., Rienstra, S. W., Mattheij, R. M. M., \& Sijtsma, P. (2011). Acoustic modes in a duct with slowly varying impedance and non-uniform mean flow and temperature. In 17th AIAA/CEAS Aeronautics Conference (Portland OR, USA June 5-8, 2011) [AIAA-2011-2871]

\section{Document status and date:}

Published: 01/01/2011

\section{Document Version:}

Publisher's PDF, also known as Version of Record (includes final page, issue and volume numbers)

\section{Please check the document version of this publication:}

- A submitted manuscript is the version of the article upon submission and before peer-review. There can be important differences between the submitted version and the official published version of record. People interested in the research are advised to contact the author for the final version of the publication, or visit the $\mathrm{DOI}$ to the publisher's website.

- The final author version and the galley proof are versions of the publication after peer review.

- The final published version features the final layout of the paper including the volume, issue and page numbers.

Link to publication

\section{General rights}

Copyright and moral rights for the publications made accessible in the public portal are retained by the authors and/or other copyright owners and it is a condition of accessing publications that users recognise and abide by the legal requirements associated with these rights.

- Users may download and print one copy of any publication from the public portal for the purpose of private study or research.

- You may not further distribute the material or use it for any profit-making activity or commercial gain

- You may freely distribute the URL identifying the publication in the public portal.

If the publication is distributed under the terms of Article 25fa of the Dutch Copyright Act, indicated by the "Taverne" license above, please follow below link for the End User Agreement:

www.tue.nl/taverne

Take down policy

If you believe that this document breaches copyright please contact us at:

openaccess@tue.nl

providing details and we will investigate your claim. 


\title{
Acoustic modes in a duct with slowly varying impedance and non-uniform mean flow and temperature
}

\author{
M. Oppeneer* W.M.J.Lazeroms ${ }^{\dagger} \quad$ S.W. Rienstra ${ }^{\ddagger} \quad$ R.M.M. Mattheij ${ }^{\S} \quad$ P. Sijtsma ${ }^{\text {If }}$ \\ NLR/TUE \\ TUE/KTH \\ $T U E$ \\ TUE \\ $N L R$
}

\begin{abstract}
Noise from the auxiliary power unit (APU) is becoming an increasingly important aircraft design constraint because of the noise exposure during ground operations ("ramp-noise"). Reduction of noise may be achieved by liners in the exhaust duct. In this paper, we will consider the propagation of sound through the APU exhaust duct, which is typically straight with an axially varying liner depth, a non-uniform mean flow and strong temperature gradients. We present a solution in the form of slowly varying modes of WKB type for the acoustic pressure field inside a duct with an impedance that is continuously varying in the axial direction. In cross-wise direction each WKB mode is given by eigenfunction-type solutions of the Pridmore-Brown equation. A new numerical approach based on a standard implementation of a collocation method supplemented by a path-following procedure is presented to solve this equation. We compare the results of the slowly-varying solution with a solution based on mode-matching between axial segments with constant impedance.
\end{abstract}

\section{Introduction}

Over the past decades, aero-acoustic research for lined flow ducts was primarily aimed at reducing the noise levels in inlet and exhaust ducts of turbo-fan engines. Since recently, however, the so-called "ramp-noise" due to the auxiliary power unit (APU) is given attention as well, as it has become a significant design constraint [1]. In this paper, we will consider the propagation of sound through the APU exhaust duct. This duct is typically straight and circular-cylindrical, and carries a non-uniform mean flow with strong temperature gradients. The acoustic impedance at the wall is axially varying due to the varying liner depth (see Figure 1).

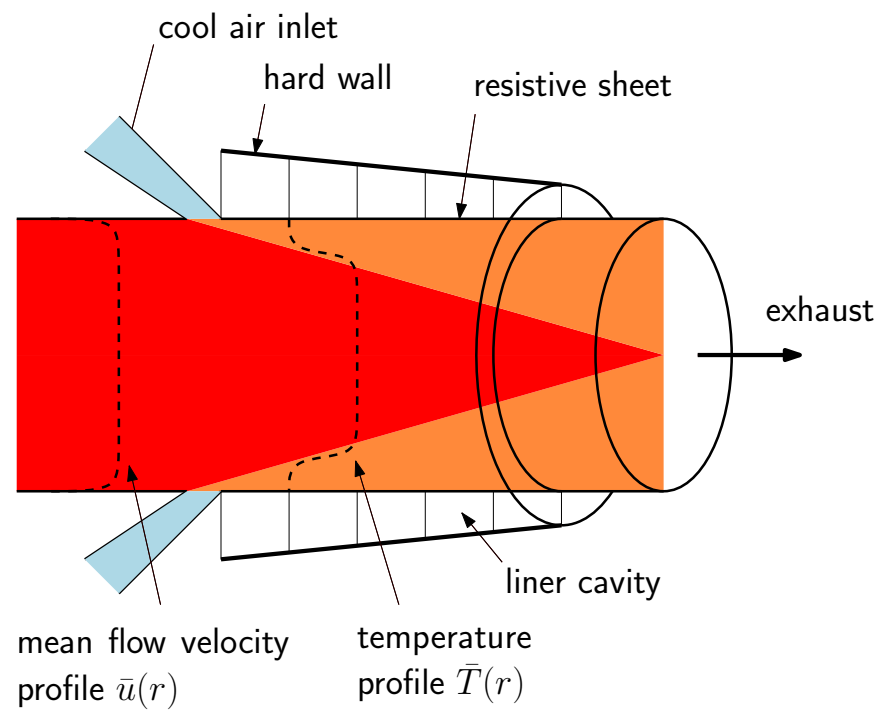

Figure 1. APU exhaust duct geometry.

*PhD student, National Aerospace Laboratory (NLR), Marknesse, The Netherlands, email: Martien.Oppeneer@nlr.nl.

${ }^{\dagger}$ PhD student, Royal Institute of Technology (KTH), Stockholm, Sweden, email: Werner@mech.kth.se.

$\ddagger$ Associate Professor, Eindhoven University of Technology (TU/e), Eindhoven, The Netherlands, Senior Member AIAA, email: S.W.Rienstra@tue.nl.

§Professor, Eindhoven University of Technology (TU/e), Eindhoven, The Netherlands, email: R.M.M.Mattheij@tue.nl.

IS Senior Scientist, National Aerospace Laboratory (NLR), Marknesse, The Netherlands, e-mail: Pieter.Sijtsma@nlr.nl.

Copyright (C) 2011 by the American Institute of Aeronautics and Astronautics, Inc. The U.S. Government has a royalty-free license to exercise all rights under the copyright claimed herein for Governmental purposes. All other rights are reserved by the copyright owner. 
To compute the sound inside the duct, we formulate the problem in terms of acoustic duct modes. These modes are solutions of the so-called Pridmore-Brown equation [2 3]. Modes exist in configurations with properties independent of the axial coordinate, i.e. ducts of constant cross-section, mean flow and wall impedance. Modes in a strict sense cease to exist if one of the problem parameters varies with the axial coordinate. However, if this variation is slow, it is possible to identify approximate solutions in the form of slowly varying "modes" by employing the WKB method (named after Wentzel, Kramers and Brillouin [4]), or method of multiple scales. This is in principle possible for any of the problem parameters [5-12], although its use is restricted by the availability of the solution of the remaining equation. In this paper we present a slowly varying mode solution for a varying impedance and otherwise constant duct and non-uniform mean flow velocity and temperature.

The cross-wise mode shape of a slowly varying mode is given by the Pridmore-Brown equation, which therefore has to be solved at each axial location. To this end we use the COLNEW [13] code, which solves boundary value problems (BVPs) in ordinary differential equations (ODEs) by collocation. By simply adding the axial wave number $k$ to the solution vector and introducing the extra equation $k^{\prime}=0$, our eigenvalue problem is easily made fit to the code.

The infinitely many solutions correspond in general with different (complex) values of $k=k_{n}$, known as the modal spectrum (we neglect here the possibility of a continuous spectrum as this is acoustically of limited importance). In order to find all (relevant) solutions, we used a path-following (or continuation) approach, where we start from an "easy" solution (for example a hard-walled duct with uniform flow) and trace the solution when the relevant problem parameter(s) is/are varied to the value(s) of interest.

Path-following has been used before for the case of uniform mean flow and a path in the impedance plane from hard wall to the impedance considered [14]. We will take into account, however, that not any path is suitable to find all modes, as has been noted in [15]. In certain directions of the complex impedance plane some modes will be missed as they appear from or disappear to infinity. In addition, our path-following approach is refined by using a prediction-correction scheme [16]. The prediction is found by linear extrapolation of the previous solutions. The correction step is then an updated solution by COLNEW, with the prediction as the starting value.

A numerical comparison will be made between some results based on the WKB approach and some results computed with a method based on mode-matching.

\section{Problem formulation}

We consider the following model for an Auxiliary Power Unit (APU) exhaust duct: a cylindrical, hollow, acoustically lined duct with radius $R$, see Figure 1 . Inside the duct, we have an inviscid, non-heat-conducting, ideal gas, described by the Euler equations

$$
\begin{aligned}
\frac{\mathrm{D} \rho}{\mathrm{D} t}+\rho \nabla \cdot \boldsymbol{v} & =0, \\
\rho \frac{\mathrm{D}}{\mathrm{D} t} \boldsymbol{v}+\nabla p & =\mathbf{0}, \\
\frac{\mathrm{D} T}{\mathrm{D} t}+(\gamma-1) T \nabla \cdot \boldsymbol{v} & =0,
\end{aligned}
$$

where $\mathrm{D} / \mathrm{D} t:=\partial / \partial t+\boldsymbol{v} \cdot \nabla$ is the total derivative, and

$$
p=\rho \mathcal{R} T,
$$

where $\mathcal{R}$ is the specific gas constant (for air: $286.73 \mathrm{~J} / \mathrm{kg} \mathrm{K}$ ) and $\gamma$ the ratio of specific heats (for air 1.402) and density, pressure, velocity and temperature are denoted by $\rho, p, v$ and $T$, respectively. The wall is treated by a locally reacting acoustic liner, which can be described for each frequency component by an impedance $Z$.

Based on a reference temperature $T_{0}$ and density $\rho_{0}$, we make the problem dimensionless by scaling on duct radius $R$, density $\rho_{0}$ and sound speed $c_{0}=\sqrt{\gamma \mathcal{R} T_{0}}$. Note that this implies that the impedance is scaled on the reference $\rho_{0} c_{0}$, rather than the more usual local $(\rho c)_{\text {wall }}$.

The total field is a superposition of a steady, parallel mean flow $\overline{\boldsymbol{v}}=\bar{u}(r) \boldsymbol{e}_{x}$ and time-harmonic acoustic perturbations, in cylindrical coordinates $(x, r, \theta)$ given by

$$
[\boldsymbol{v}, \rho, p, T](x, r, \theta, t)=[\overline{\boldsymbol{v}}, \bar{\rho}, \bar{p}, \bar{T}](r)+\operatorname{Re}([\tilde{\boldsymbol{v}}, \tilde{\rho}, \tilde{p}, \tilde{T}](x, r) \exp (-\mathrm{i} \omega t+\mathrm{i} m \theta)),
$$

where $\omega$ and $m$ are the non-dimensional frequency and the circumferential wavenumber respectively. The mean flow properties are taken to be independent of $x$, which is possible because of the assumed inviscid and non-heatconducting medium. The wall impedance can depend on $x$, but influences only the acoustic field. Consequently, the mean flow quantities depend only on $r$ :

$$
\overline{\boldsymbol{v}}=\bar{u}(r) \boldsymbol{e}_{x}, \quad \bar{T}=\bar{T}(r), \quad \bar{p}=p_{0}=\frac{1}{\gamma}, \quad \bar{\rho}(r)=\frac{1}{\bar{T}(r)} .
$$


Substituting (2) into (1) and linearizing yields the following system of equations for the perturbations

$$
\begin{aligned}
&\left(-\mathrm{i} \omega+\bar{u} \frac{\partial}{\partial x}\right) \tilde{\rho}+\frac{1}{r} \frac{\partial(r \bar{\rho} \tilde{v})}{\partial r}+\bar{\rho}\left(\frac{\mathrm{i} m}{r} \tilde{w}+\frac{\partial \tilde{u}}{\partial x}\right)=0, \\
&\left(-\mathrm{i} \omega+\bar{u} \frac{\partial}{\partial x}\right) \tilde{u}+\tilde{v} \frac{\mathrm{d} \bar{u}}{\mathrm{~d} r}+\frac{1}{\bar{\rho}} \frac{\partial \tilde{p}}{\partial x}=0, \\
&\left(-\mathrm{i} \omega+\bar{u} \frac{\partial}{\partial x}\right) \tilde{v}+\frac{1}{\bar{\rho}} \frac{\partial \tilde{p}}{\partial r}=0, \\
&\left(-\mathrm{i} \omega+\bar{u} \frac{\partial}{\partial x}\right) \tilde{w}+\frac{\mathrm{i} m}{r \bar{\rho}} \tilde{p}=0, \\
&\left(-\mathrm{i} \omega+\bar{u} \frac{\partial}{\partial x}\right) \bar{T}+\frac{\mathrm{d} \bar{T}}{\mathrm{~d} r} \tilde{v}+(\gamma-1) \bar{T}\left(\frac{1}{r} \frac{\partial}{\partial r}(r \tilde{v})+\frac{\mathrm{i} m}{r} \tilde{w}+\frac{\partial \tilde{u}}{\partial x}\right)=0, \\
& \gamma \tilde{p}=\bar{\rho} \tilde{T}+\tilde{\rho} \bar{T} .
\end{aligned}
$$

At the impedance wall, we use the Ingard-Myers [17, 18] boundary condition, which describes the effect of the thin boundary layer on the impedance.

$$
-\mathrm{i} \omega \tilde{v}=\left(-\mathrm{i} \omega+\bar{u} \frac{\partial}{\partial x}\right)\left(\frac{\tilde{p}}{Z}\right), \quad \text { at } r=1 .
$$

\section{Pridmore-Brown equation for constant impedance}

First, we consider a duct with a constant impedance along the wall, so the problem becomes invariant in the axial direction. We introduce acoustic modes of the form

$$
[\tilde{u}, \tilde{v}, \tilde{w}, \tilde{\rho}, \tilde{p}, \tilde{T}](r)=[U, V, W, D, P, T](r) \exp (+\mathrm{i} k x),
$$

where $k$ is the axial modal wavenumber, which is to be found together with the modal shape function. When (6) is substituted in (4), we have

$$
\begin{aligned}
&-\mathrm{i}(\omega-k \bar{u}) D+\frac{1}{r} \frac{\mathrm{d}(r \bar{\rho} V)}{\mathrm{d} r}+\bar{\rho}\left(\frac{\mathrm{i} m}{r} W+\mathrm{i} k U\right)=0, \\
&-\mathrm{i}(\omega-k \bar{u}) U+V \frac{\mathrm{d} \bar{u}}{\mathrm{~d} r}+\frac{1}{\bar{\rho}} \mathrm{i} k P=0, \\
&-\mathrm{i}(\omega-k \bar{u}) V+\frac{1}{\bar{\rho}} \frac{\mathrm{d} P}{\mathrm{~d} r}=0, \\
&-\mathrm{i}(\omega-k \bar{u}) W+\frac{\mathrm{i} m}{r \bar{\rho}} P=0, \\
&-\mathrm{i}(\omega-k \bar{u}) \bar{T}+\frac{\mathrm{d} \bar{T}}{\mathrm{~d} r} V+(\gamma-1) \bar{T}\left(\frac{1}{r} \frac{\mathrm{d}}{\mathrm{d} r}(r V)+\frac{\mathrm{i} m}{r} W+\mathrm{i} k U\right)=0, \\
& \gamma P=\bar{\rho} T+D \bar{T} .
\end{aligned}
$$

This system can be reduced further to a single ordinary differential equation for the pressure perturbation amplitude $P$, yielding the Pridmore-Brown [2] equation

$$
P^{\prime \prime}+\left[\frac{1}{r}+\frac{2 k \bar{u}^{\prime}}{\omega-k \bar{u}}+\frac{\bar{T}^{\prime}}{\bar{T}}\right] P^{\prime}+\left[\frac{(\omega-k \bar{u})^{2}}{\bar{T}}-k^{2}-\frac{m^{2}}{r^{2}}\right] P=0 \quad \text { on } 0<r \leqslant 1,
$$

where the prime is used to denote differentiation with respect to $r$. For uniform flow $\bar{u}=M_{0}$ and constant temperature $\bar{T}=1,(8)$ reduces to Bessel's equation, with solution

$$
P(r)=A J_{m}(\alpha r)+B Y_{m}(\alpha r), \quad \alpha^{2}=\left(\omega-k M_{0}\right)^{2}-k^{2} .
$$

The boundary condition (5) becomes

$$
P^{\prime}-\left[\frac{\mathrm{i}(\omega-k \bar{u})^{2}}{\omega Z \bar{T}}\right] P=0 \quad \text { at } r=1 .
$$

Equation (8) has solutions that are singular at $r=0$. To enforce regular solutions, we add the boundary condition

$$
\begin{aligned}
& P^{\prime}=0 \quad \text { at } r=0, \quad \text { for } m \neq 1 \text {, } \\
& P=0 \quad \text { at } r=0, \quad \text { for } m=1 \text {. }
\end{aligned}
$$


Otherwise, $P$ is determined up to a multiplicative constant. For each $m$, equation (8) has multiple solutions, denoted by

$$
P=A \psi_{m n}(r) \text {. }
$$

Each solution is characterized by the eigenfunction $\psi_{m n}(r)$ and the corresponding wavenumber ${ }^{1} k_{m n}$. Left and right-running waves will be distinguished by the sign of the imaginary part of $k_{m n}$, or by taking the suitable limit in $Z$ if $\operatorname{Im}\left(k_{m n}\right)=0$. In rare cases the mode may be an instability with $k_{m n}$ belonging to the other complex half plane [15, 19], but we will not consider this here.

\section{III.A. A high-frequency approximation (radial WKB approximation of modes)}

Since non-trivial solutions of (8) are unknown, or at least rare, it is useful to consider approximations. Following the WKB-type method described in [3], we can find some approximate solutions for high frequency $\omega$. If we write

$$
P^{\prime \prime}+\beta(r, k) P^{\prime}+\gamma(r, k) P=0
$$

and note that $\beta=O(1)$ and $\gamma=O\left(\omega^{2}\right)$ when $\omega \rightarrow \infty$, we can make the WKB assumption

$$
P(r) \simeq A(r) \exp \left(\mathrm{i} \int^{r} \xi\left(r^{\prime}\right) \mathrm{d} r^{\prime}\right) .
$$

After sorting terms of equal order of magnitude, we find in the usual way the approximate equation

$$
\left(\gamma-\xi^{2}\right) A+\mathrm{i}\left(\xi^{\prime} A+2 \xi A^{\prime}+\xi \beta A\right)+\cdots=0 .
$$

This has solutions

$$
\xi= \pm \sqrt{\gamma}, \quad A=\frac{\omega-k \bar{u}}{\gamma^{1 / 4} \sqrt{r \bar{T}}} .
$$

The resulting (not necessarily uniformly valid) solutions

$$
P=\frac{\omega-k \bar{u}}{\gamma^{1 / 4 \sqrt{r \bar{T}}}} \exp \left( \pm \mathrm{i} \int^{r} \sqrt{\gamma(s)} \mathrm{d} s\right)
$$

reveal the important information that acoustic solutions, where $k$ is real or nearly real, are practically only important along that part of the $r$-interval where $\gamma(r)>0$, and otherwise exponentially small. (Zeros of $\gamma$ are known as turning points [5].) Generally, $\gamma$ is negative near $r=0$ when $m \neq 0$ (yielding the $P \propto r^{m}$ behaviour near $r=0$ ), with a zero at say $r_{1}$. Although not as general, this may also happen near $r=1$, with a zero at say $r_{2}$, depending on details of the velocity and temperature profiles. If this is the case and the solution is exponentially small near $r=1$, the effect of the boundary condition is negligible, and the solution is practically independent of the wall impedance. The wave numbers $k$ are then found from a "quantization condition" [20] that requires that an integer number of radial semi-wavelengths has to fit between the turning points $r_{1}$ and $r_{2}$, with on each side an extra $\frac{1}{4} \pi$ due to the required matching through the turning point to the decaying field [5]. If $m \neq 0$ (and $\gamma$ has no more zeros than $r_{1}$ and $r_{2}$ ) this condition is

$$
\int_{r_{1}}^{r_{2}} \sqrt{\gamma(r, k)} \mathrm{d} r=\left(n-\frac{1}{2}\right) \pi, \quad n=1,2, \ldots
$$

If $m=0$ with only one turning point, we have

$$
\int_{0}^{r_{2}} \sqrt{\gamma(r, k)} \mathrm{d} r=\left(n-\frac{1}{4}\right) \pi, \quad n=1,2, \ldots
$$

\section{Mode-matching for piecewise constant impedance}

As a reference case, we consider a duct that is divided in axial segments, each having a constant impedance. For each segment, the acoustic pressure is governed by the Pridmore-Brown equation, as discussed previously. For the segmented case, we assume uniform mean flow and constant temperature, so the Pridmore-Brown equation reduces to Bessel's equation, which has analytical solutions. To compute the field inside the entire duct, we relate the modes in adjacent segments by continuity of pressure and axial velocity, the so-called mode-matching method. We describe the approach that was implemented in the NLR code BAHAMAS, which is an extension of the approach described in Reference 21 to multiple lined sections.

\footnotetext{
${ }^{1}$ The axial modal wavenumber $k_{m n}$ is not the actual eigenvalue, but directly depends on it.
} 
Consider two segments I and II, left and right of an interface (see Figure 2), where segment I has a hard wall and segment II has an impedance wall. The total field for a given circumferential wavenumber $m$ in each segment is a superposition of all (in practice limited to $n_{\max }$ ) modes

$$
\begin{gathered}
p^{I}(x, r, \theta)=\sum_{n=1}^{\infty}\left(A_{m n}^{I} \varphi_{m n}^{+}(r) \mathrm{e}^{\mathrm{i} \kappa_{m n}^{+} x}+B_{m n}^{I} \varphi_{m n}^{-}(r) \mathrm{e}^{\mathrm{i} \kappa_{m n}^{-} x}\right), \\
p^{I I}(x, r, \theta)=\sum_{n=1}^{\infty}\left(A_{m n}^{I I} \psi_{m n}^{+}(r) \mathrm{e}^{\mathrm{i} k_{m n}^{+} x}+B_{m n}^{I I} \psi_{m n}^{-}(r) \mathrm{e}^{\mathrm{i} k_{m n}^{-} x}\right),
\end{gathered}
$$

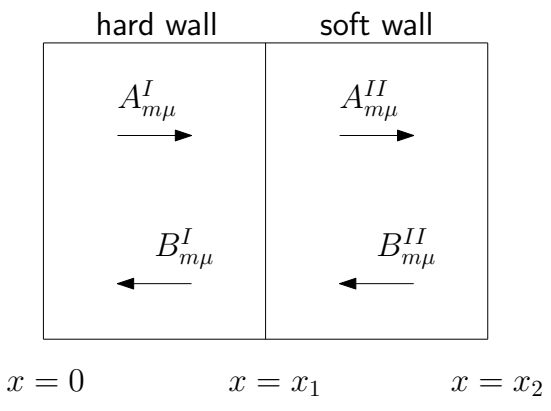

Figure 2. Mode matching geometry.

where $A$ and $B$ are the amplitudes of the right and left running modes in each segment, respectively. In the hardwalled sections the left and right running mode shapes are the same, so $\varphi_{m n}^{+}=\varphi_{m n}^{-}$. Continuity of pressure and axial velocity at each interface and the subsequent projection on the set of hard-wall modes yields a linear system for the amplitudes

$$
\left[\begin{array}{cc}
\mathbf{c}^{+} & -\mathbf{a}^{-} \\
\mathbf{d}^{+} & -\mathbf{b}^{-}
\end{array}\right]\left[\begin{array}{c}
\mathbf{A}^{I I} \\
\mathbf{B}^{I}
\end{array}\right]=\left[\begin{array}{ll}
\mathbf{a}^{+} & -\mathbf{c}^{-} \\
\mathbf{b}^{+} & -\mathbf{d}^{-}
\end{array}\right]\left[\begin{array}{c}
\mathbf{A}^{I} \\
\mathbf{B}^{I I}
\end{array}\right] .
$$

In this system, the modal amplitude vectors $\mathbf{A}^{I I}$ and $\mathbf{B}^{I}$ for the outgoing waves are computed from the amplitude vectors $\mathbf{A}^{I}$ and $\mathbf{B}^{I I}$ of the incident waves. The $n_{\max } \times n_{\max }$ sized matrices $\mathbf{a}, \mathbf{b}, \mathbf{c}, \mathbf{d}$ are based on overlap integrals of Bessel functions. The combined transmissions and reflections at all interfaces give the total field in the entire duct.

\section{Slowly varying modes for slowly varying impedance (axial WKB approximation)}

If the impedance varies in the axial direction, there are strictly speaking no modes possible anymore. However, if we assume that the inherent length scale $L$ of typical variations of $Z(\hat{x} / L)$ (where $\hat{x}=R x$ is the dimensional axial coordinate) is large compared to the duct radius $R$, i.e.

$$
Z\left(\frac{\hat{x}}{L}\right)=Z\left(\frac{R}{L} x\right)=Z(\varepsilon x)=Z(X), \quad \varepsilon=\frac{R}{L} \ll 1, \quad X=\varepsilon x,
$$

we can use this small parameter $\varepsilon$ to construct so-called slowly varying modes by a variant of the WKB method. Assuming that the modal wavenumbers are typically equal or larger than $O(1)$, we approximate the acoustic field by modes of which the amplitude, mode shape and modal wavenumber vary only slowly in the axial direction. We assume modal solutions of the form

$$
[\tilde{u}, \tilde{v}, \tilde{w}, \tilde{\rho}, \tilde{p}, \tilde{T}](r, X)=[U, V, W, D, P, T](r, X) \exp \left(\frac{\mathrm{i}}{\varepsilon} \int_{0}^{X} \mu(\eta) \mathrm{d} \eta\right),
$$

where $\mu(X)$ is the axial wavenumber depending on the slow coordinate $X$. This is the so-called WKB assumption.

Substituting (17) into (4) yields

$$
\begin{aligned}
& -\mathrm{i} \Lambda D+\varepsilon \bar{u} \frac{\partial D}{\partial X}+\frac{1}{r} \frac{\partial}{\partial r}(r \bar{\rho} V)+\bar{\rho}\left(\frac{\mathrm{i} m}{r} W+\varepsilon \frac{\partial U}{\partial X}+\mathrm{i} \mu U\right)=0, \\
& -\mathrm{i} \Lambda U+\varepsilon \bar{u} \frac{\partial U}{\partial X}+\frac{\mathrm{d} \bar{u}}{\mathrm{~d} r} V+\frac{1}{\bar{\rho}}\left(\varepsilon \frac{\partial P}{\partial X}+\mathrm{i} \mu P\right)=0, \\
& -\mathrm{i} \Lambda V+\varepsilon \bar{u} \frac{\partial V}{\partial X}+\frac{1}{\bar{\rho}} \frac{\partial P}{\partial r}=0, \\
& -\mathrm{i} \Lambda W+\varepsilon \bar{u} \frac{\partial W}{\partial X}+\frac{\mathrm{i} m}{r \bar{\rho}} P=0, \\
& -\mathrm{i} \Lambda T+\varepsilon \bar{u} \frac{\partial T}{\partial X}+\frac{\mathrm{d} \bar{T}}{\mathrm{~d} r} V+(\gamma-1) \bar{T}\left[\frac{1}{r} \frac{\partial}{\partial r}(r V)+\frac{\mathrm{i} m}{r} W+\varepsilon \frac{\partial U}{\partial X}+\mathrm{i} \mu U\right]=0, \\
& \gamma P=\bar{\rho} T+\bar{T} D
\end{aligned}
$$

with $\Lambda:=\omega-\mu \bar{u}$. The corresponding boundary condition obtained from (5) is

$$
-\mathrm{i} \omega V=-\frac{\mathrm{i} \Lambda}{Z} P+\varepsilon \bar{u} \frac{\partial}{\partial X}\left(\frac{P}{Z}\right), \quad \text { at } r=1 .
$$


Expand the amplitude functions in $\varepsilon$ as follows ${ }^{2}$

$$
[U, V, W, D, P, T](r, X ; \varepsilon)=\left[U_{0}, V_{0}, W_{0}, D_{0}, P_{0}, T_{0}\right](r, X)+\varepsilon\left[U_{1}, V_{1}, W_{1}, D_{1}, P_{1}, T_{1}\right](r, X)+O\left(\varepsilon^{2}\right) .
$$

The leading order equations obtained after substitution of this expansion in equations (18) are

$$
\begin{aligned}
-\mathrm{i} \Lambda D_{0}+\frac{1}{r} \frac{\partial}{\partial r}\left(r \bar{\rho} V_{0}\right)+\bar{\rho}\left(\frac{\mathrm{i} m}{r} W_{0}+\mathrm{i} \mu U_{0}\right) & =0, \\
-\mathrm{i} \Lambda U_{0}+\frac{\mathrm{d} \bar{u}}{\mathrm{~d} r} V_{0}+\frac{\mathrm{i} \mu}{\bar{\rho}} P_{0} & =0, \\
-\mathrm{i} \Lambda V_{0}+\frac{1}{\bar{\rho}} \frac{\partial P_{0}}{\partial r} & =0, \\
-\mathrm{i} \Lambda W_{0}+\frac{\mathrm{i} m}{r \bar{\rho}} P_{0} & =0, \\
-\mathrm{i} \Lambda T_{0}+\frac{\mathrm{d} \bar{T}}{\mathrm{~d} r} V_{0}+(\gamma-1) \bar{T}\left[\frac{1}{r} \frac{\partial}{\partial r}\left(r V_{0}\right)+\frac{\mathrm{i} m}{r} W_{0}+\mathrm{i} \mu U_{0}\right] & =0, \\
\gamma P_{0} & =\bar{\rho} T_{0}+\bar{T} D_{0},
\end{aligned}
$$

with the boundary condition

$$
-\mathrm{i} \omega V_{0}=-\frac{\mathrm{i} \Lambda}{Z} P_{0}, \quad \text { at } r=1 .
$$

This system of equations has the same structure as (7), the only difference being the dependence on $X$, which serves as a parameter. Therefore, we have effectively the same equation as $(8)$ for the pressure $P_{0}$

$$
\mathcal{L} P_{0}:=\left[\frac{\partial^{2}}{\partial r^{2}}+\left(\frac{1}{r}-\frac{2}{\Lambda} \frac{\partial \Lambda}{\partial r}+\frac{\bar{T}^{\prime}}{\bar{T}}\right) \frac{\partial}{\partial r}+\left(\frac{\Lambda^{2}}{\bar{T}}-\mu^{2}-\frac{m^{2}}{r^{2}}\right)\right] P_{0}=0,
$$

a regularity condition at $r=0$ and a boundary condition

$$
\frac{\partial P_{0}}{\partial r}-\frac{\mathrm{i} \Lambda^{2} \bar{\rho}}{\omega Z} P_{0}=0, \quad \text { at } r=1 .
$$

For every fixed $X$, this problem is exactly the same as the constant impedance problem described by $(8)$ and $(9)$. The general solution will be of the form

$$
P_{0}(r, X)=N(X) \psi_{m n}(r, X)
$$

where $\psi_{m n}$ is again an eigenfunction, essentially the same as $\psi_{m n}$ of (10), this time parametrically depending on $X$, and $N(X)$ is a slowly varying amplitude function that is still unknown. As before, solving for $\psi_{m n}$ includes the axial wavenumber $\mu_{m n}$ as a function of $X$ for any given $\omega$ and $m$.

To determine $N(X)$, the first order equations obtained from (18) after the expansion in (19) are needed. We do not have to solve them, but we need minimal conditions for the existence of the solution. These equations are

$$
\begin{aligned}
-\mathrm{i} \Lambda D_{1}+\frac{1}{r} \frac{\partial}{\partial r}\left(r \bar{\rho} V_{1}\right)+\bar{\rho}\left(\frac{\mathrm{i} m}{r} W_{1}+\mathrm{i} \mu U_{1}\right) & =-\left(\bar{u} \frac{\partial D_{0}}{\partial X}+\bar{\rho} \frac{\partial U_{0}}{\partial X}\right), \\
-\mathrm{i} \Lambda U_{1}+\frac{\mathrm{d} \bar{u}}{\mathrm{~d} r} V_{1}+\frac{\mathrm{i} \mu}{\bar{\rho}} P_{1} & =-\left(\bar{u} \frac{\partial U_{0}}{\partial X}+\frac{1}{\bar{\rho}} \frac{\partial P_{0}}{\partial X}\right), \\
-\mathrm{i} \Lambda V_{1}+\frac{1}{\bar{\rho}} \frac{\partial P_{1}}{\partial r} & =-\bar{u} \frac{\partial V_{0}}{\partial X}, \\
-\mathrm{i} \Lambda W_{1}+\frac{\mathrm{i} m}{r \bar{\rho}} P_{1} & =-\bar{u} \frac{\partial W_{0}}{\partial X}, \\
-\mathrm{i} \Lambda T_{1}+\frac{\mathrm{d} \bar{T}}{\mathrm{~d} r} V_{1}+(\gamma-1) \bar{T}\left[\frac{1}{r} \frac{\partial}{\partial r}\left(r V_{1}\right)+\frac{\mathrm{i} m}{r} W_{1}+\mathrm{i} \mu U_{1}\right] & =-\left[\bar{u} \frac{\partial T_{0}}{\partial X}+(\gamma-1) \bar{T} \frac{\partial U_{0}}{\partial X}\right], \\
\gamma P_{1} & =\bar{\rho} T_{1}+\bar{T} D_{1},
\end{aligned}
$$

with regularity condition at $r=0$ and boundary condition

$$
-\mathrm{i} \omega V_{1}+\frac{\mathrm{i} \Lambda}{Z} P_{1}=\bar{u} \frac{\partial}{\partial X}\left(\frac{P_{0}}{Z}\right) \quad \text { at } r=1 .
$$

\footnotetext{
${ }^{2}$ Note that the subscript 0 is not to be confused with the subscript for reference value.
} 
In a similar way as before, we can find a single equation for $P_{1}$ with a right-hand side that only contains $P_{0}$. The result after some laborious calculations is

$$
\mathcal{L} P_{1}=\mathcal{F}:=-\frac{2 \mathrm{i} \bar{u}}{\Lambda} \frac{\partial}{\partial X}\left[\frac{\partial}{\partial r} \ln \left(\frac{\Lambda}{\bar{u}}\right) \frac{\partial P_{0}}{\partial r}\right]-\mathrm{i} \frac{\bar{u}}{P_{0}} \frac{\partial}{\partial X}\left[\left(\frac{\Lambda}{\bar{T}}+\frac{\mu}{\bar{u}}\right) P_{0}^{2}\right],
$$

with

$$
\frac{\partial P_{1}}{\partial r}-\frac{\mathrm{i} \Lambda^{2} \bar{\rho}}{\omega Z} P_{1}=-\frac{\bar{u} \bar{\rho} Z}{\omega P_{0}} \frac{\partial}{\partial X}\left(\frac{\Lambda P_{0}^{2}}{Z^{2}}\right), \quad \text { at } r=1 .
$$

Instead of solving for $P_{1}$, which would lead to yet another undetermined factor, we are going to derive a solvability condition for (24) that only contains leading order information. This is achieved by multiplying (24a) by $\bar{T} P_{0} / \Lambda^{2}$, (21a) by $\bar{T} P_{1} / \Lambda^{2}$, subtracting the two equations and integrating the result. This gives

$$
\int_{0}^{1} \frac{\bar{T}}{\Lambda^{2}}\left(P_{0} \mathcal{L} P_{1}-P_{1} \mathcal{L} P_{0}\right) r \mathrm{~d} r=\int_{0}^{1} \frac{\bar{T}}{\Lambda^{2}} P_{0} \mathcal{F} r \mathrm{~d} r
$$

One can show that the operator $\left(r \bar{T} / \Lambda^{2}\right) \mathcal{L}$ is self-adjoint, so that the left-hand side of (25) can be reduced to a term containing only boundary data, i.e.

$$
\begin{gathered}
\int_{0}^{1} \frac{\bar{T}}{\Lambda^{2}}\left(P_{0} \mathcal{L} P_{1}-P_{1} \mathcal{L} P_{0}\right) r \mathrm{~d} r=\int_{0}^{1} \frac{\partial}{\partial r}\left[\frac{r \bar{T}}{\Lambda^{2}}\left(P_{0} \frac{\partial P_{1}}{\partial r}-P_{1} \frac{\partial P_{0}}{\partial r}\right)\right] \mathrm{d} r= \\
{\left[\frac{r \bar{T}}{\Lambda^{2}}\left(P_{0} \frac{\partial P_{1}}{\partial r}-P_{1} \frac{\partial P_{0}}{\partial r}\right)\right]_{r=0}^{r=1}=\left.\frac{\bar{T}}{\Lambda^{2}}\left(P_{0} \frac{\partial P_{1}}{\partial r}-P_{1} \frac{\partial P_{0}}{\partial r}\right)\right|_{r=1}=-\left.\frac{\bar{u} Z}{\omega \Lambda^{2}} \frac{\partial}{\partial X}\left(\frac{\Lambda P_{0}^{2}}{Z^{2}}\right)\right|_{r=1}}
\end{gathered}
$$

where we also used the boundary conditions $(21 b)$ and $(24 b)$. Using this result together with $(24 a)$ in $(25)$ then leads to the following solvability condition for the first order problem

$$
\mathrm{i} \int_{0}^{1}\left\{\frac{2 \bar{u} \bar{T}}{\Lambda^{3}} P_{0} \frac{\partial}{\partial X}\left[\frac{\partial}{\partial r} \ln \left(\frac{\Lambda}{\bar{u}}\right) \frac{\partial P_{0}}{\partial r}\right]+\frac{\bar{u} \bar{T}}{\Lambda^{2}} \frac{\partial}{\partial X}\left[\left(\frac{\Lambda}{\bar{T}}+\frac{\mu}{\bar{u}}\right) P_{0}^{2}\right]\right\} r \mathrm{~d} r-\left.\frac{\bar{u} Z}{\omega \Lambda^{2}} \frac{\partial}{\partial X}\left(\frac{\Lambda P_{0}^{2}}{Z^{2}}\right)\right|_{r=1}=0
$$

The next step is to substitute the general solution of $P_{0}$ shown in (22) into (27). After working out and rearranging terms, we arrive at a first order equation for the amplitude function $N$

$$
g(X) \frac{\mathrm{d} N^{2}}{\mathrm{~d} X}=-f(X) N^{2}(X)
$$

with (in principle known) functions

$$
\begin{aligned}
& f(X)=\mathrm{i} \int_{0}^{1}\left\{\frac{2 \psi}{\Lambda^{3}} \frac{\partial}{\partial X}\left[\bar{u} \bar{T} \frac{\partial}{\partial r} \ln \left(\frac{\Lambda}{\bar{u}}\right) \frac{\partial \psi}{\partial r}\right]+\frac{1}{\Lambda^{2}} \frac{\partial}{\partial X}\left[(\bar{u} \Lambda+\mu \bar{T}) \psi^{2}\right]\right\} r \mathrm{~d} r-\left.\frac{\bar{u} Z}{\omega \Lambda^{2}} \frac{\partial}{\partial X}\left(\frac{\Lambda \psi^{2}}{Z^{2}}\right)\right|_{r=1}, \\
& g(X)=\mathrm{i} \int_{0}^{1}\left\{\frac{\psi}{\Lambda^{3}} \bar{u} \bar{T} \frac{\partial}{\partial r} \ln \left(\frac{\Lambda}{\bar{u}}\right) \frac{\partial \psi}{\partial r}+\frac{\bar{u} \Lambda+\mu \bar{T}}{\Lambda^{2}} \psi^{2}\right\} r \mathrm{~d} r-\left.\frac{\bar{u}}{\omega \Lambda Z} \psi^{2}\right|_{r=1} .
\end{aligned}
$$

This equation has the solution

$$
N^{2}=N_{0}^{2} \exp \left(-\int_{0}^{X} \frac{f(\eta)}{g(\eta)} \mathrm{d} \eta\right)
$$

where $N_{0}$ is a normalization constant that can be determined from the source, e.g. at the beginning of the duct.

In principle, (22) can now be computed, provided the partial derivatives $\partial \mu(X) / \partial X$ and $\partial \psi(r, X) / \partial X$ are known. Since $\mu(X)$ and $\psi(r, X)$ are only known from the solution of the Pridmore-Brown equation, which has to be solved numerically, the $X$-derivatives have to be computed numerically as well. have

The results can be simplified for some special cases. For uniform mean flow and arbitrary temperature we

$$
\frac{f(X)}{g(X)}=\frac{\frac{\partial a(X)}{\partial X}-\left[\frac{\mathrm{i} \bar{u} \Lambda}{\omega Z^{2}} \psi^{2} \frac{\mathrm{d} Z}{\mathrm{~d} X}\right]_{r=1}}{a(X)}, \quad \text { where } a(X)=\int_{0}^{1}(\bar{u} \Lambda+\mu \bar{T}) \psi^{2} r \mathrm{~d} r+\left[\frac{\mathrm{i} \bar{u} \Lambda}{\omega Z} \psi^{2}\right]_{r=1},
$$

such that 29 is reduced to

$$
N^{2}(X)=\frac{N_{0}^{2}}{a(X)} \exp \left(-\int^{X} \frac{h(\eta)}{a(\eta)} \mathrm{d} \eta\right), \quad \text { where } \quad h(X)=\left[\frac{\mathrm{i} \bar{u} \Lambda}{\omega Z^{2}} \psi^{2} \frac{\mathrm{d} Z}{\mathrm{~d} X}\right]_{r=1} .
$$


For zero mean flow and arbitrary temperature, this can be reduced even further, to

$$
N^{2}(X)=N_{0}^{2}\left(\mu \int_{0}^{1} \bar{T} \psi^{2} r \mathrm{~d} r\right)^{-1} .
$$

The result in (29) may be compared with the one given by [9], where the duct varies in diameter and the mean flow includes swirl, but the impedance is taken constant. With the APU geometry in mind of a constant duct but varying impedance, we could obtain a simpler result, that is relatively easily applicable when numerical solutions of the Pridmore-Brown equation are available.

\section{Numerical approach: COLNEW and path-following}

As discussed above, the Pridmore-Brown equation describes an eigenvalue problem with infinitely many solutions. In case of a hard wall, uniform mean flow and constant temperature, the classical analytical solutions exist in the form of Bessel functions (section III), while the eigenvalues are given through the easily found (because real) zeros of the derivatives of the Bessel function. For most other cases solutions have to be found entirely numerically.

Among realized methods to solve the BVP are the direct shooting method [22] and various collocation methods [7. 9 23,24]. The advantage of a shooting method is obviously its simplicity, but care is required when the solution is zero or very small at both ends of the interval. Therefore, also here a collocation method is chosen, viz. the standard implementation COLNEW [13, 25], which is freely available from Netlib [26]. This code is capable of solving mixed-order systems of BVPs in ODEs. It is based on collocation at Gaussian points using a Runge-Kutta monomial basis representation, it uses a damped Newton solver to solve the system of non-linear equations and it has automatic meshing.

We first put the eigenvalue problem in a form suitable for a standard BVP solver by adding the axial wave number $k$ to the solution vector, introducing the extra equation $k^{\prime}=0$, adding a normalizing boundary condition like $P(1)=1$, and splitting the equations in real and imaginary parts.

Then we make sure to have suitable starting guesses. As an eigenvalue problem, the system of equations to be solved is non-linear. As with any non-linear problem it is important to start with a good initial guess. This is particularly important if we want to make sure to find all (i.e. all physically relevant) solutions (modes) and corresponding eigenvalues (modal wave numbers). This is realized by a path-following (or continuation) approach, where we start from an "easy" solution (in our case a hard-walled duct with uniform mean flow velocity and temperature) and trace the solution when the relevant problem parameters are varied until they reach their target values. Essentially, we embed the problem in a formulation with a continuation parameter $\lambda$. Path-following can thus be seen as an evolution problem [14,16] with the problem evolving from a known solution for $\lambda=0$ to the target solution at $\lambda=1$.

Apart from making sure that our calculation converges, that we can find all solutions, and that we can investigate the behaviour of a solution as a function of a parameter, there is another advantage of path-following. When we are interested in a series of $\lambda$-values of the same continuation, for example when performing parameter scans, it is very efficient to save intermediate solutions when they are passed along the way.

To compute the relevant solution with mean flow velocity $M(r)$, temperature $T(r)$ and impedance $Z$ starting from the analytical solution for uniform mean flow with Mach number $M_{0}$, uniform temperature and an (almost) hard-wall impedance $Z_{\infty}$ we use the embedding

$$
\begin{aligned}
\bar{u}(r) & =(1-\lambda) M_{0}+\lambda M(r), \\
\bar{T}(r) & =(1-\lambda)+\lambda T(r), \\
Z & =(1-\lambda) Z_{\infty}+\lambda Z .
\end{aligned}
$$

(Other continuation parameterizations are also possible, but this was found to work generally well.) We vary the impedance along a vertical trajectory in the complex plane (up or downwards, depending on the mean flow Mach number) in order to have certainty about finding all possible surface modes [15]. The impedance, mean flow velocity and temperature are gradually changed to their desired non-uniform profiles in parallel, or one after another.

Since each solution of the Pridmore-Brown equation is characterized by an eigenfunction and an axial wavenumber $k$, we can trace $k(\lambda)$ as a curve in the complex plane. To determine the number of intermediate solutions and the corresponding values of $\lambda$ we use a prediction-correction scheme [16] (see Figure 3). We use linear extrapolation of two previously computed $k$-values for the prediction step. In general, the prediction will not satisfy the Pridmore-Brown equation. The prediction is therefore corrected subsequently.

We trace the eigenvalue $k=k(\lambda)$ and solution vector $\mathbf{u}=\mathbf{u}(\lambda)$ for $\lambda \in[0,1]$ using steps $h_{j}:=\lambda_{j}-\lambda_{j-1}$. 
From two previous solutions $k_{j-2}=k\left(\lambda_{j-2}\right)$ and $k_{j-1}=k\left(\lambda_{j-1}\right)$ we predict a value for $k_{j}$ by linear extrapolation

$$
\tilde{k}_{j}=k_{j-1}+h_{j} \frac{k_{j-1}-k_{j-2}}{h_{j-1}} .
$$

The prediction of $\mathbf{u}$ is performed similarly. By substituting $\lambda_{j-1}$ and $\lambda_{j-2}$ in the Taylor series expansion of $k(\lambda)$ around $\lambda=\lambda_{j}$ we find expressions for $k_{j-1}$ and $k_{j-2}$ that can be used with (34) to find

$$
\tilde{k}_{j}=k_{j}-\frac{1}{2} h_{j}\left(h_{j}+h_{j-1}\right) k_{j}^{\prime \prime}+\cdots .
$$

Thus the error $\epsilon_{j}$ between the exact and predicted value is

$$
\epsilon_{j}=\left|k_{j}-\tilde{k}_{j}\right|=\left|\frac{1}{2} h_{j}\left(h_{j}+h_{j-1}\right) k_{j}^{\prime \prime}+\cdots\right|=c h_{j}^{2},
$$

where $c$ (i.e. $\sim k_{j}^{\prime \prime}$ ) is assumed to be sufficiently smooth. Given this $j$-th step error, we can compute the next step size $h_{j+1}$ such that the error remains around some tolerance $\epsilon_{t o l}=c h_{j+1}^{2}$. This leads to

$$
h_{j+1}=h_{j} \frac{h_{j+1}}{h_{j}}=h_{j} \sqrt{\frac{c h_{j+1}^{2}}{c h_{j}^{2}}}=h_{j} \sqrt{\frac{\epsilon_{t o l}}{\epsilon_{j}}} .
$$

This tolerance level is selected as follows. The left and right running hard-wall uniform-flow axial wavenumbers $\hat{k}$ of circumferential order $m$ and radial order $n$ are given by

$$
\hat{k}_{m n}=\frac{-\omega M_{0} \pm \sqrt{\omega^{2}-\alpha_{m n}^{2}\left(1-M_{0}^{2}\right)}}{1-M_{0}^{2}},
$$

with $\alpha_{m n}$ the $n$-th zero $j_{m n}^{\prime}$ of the derivative of Besselfunction $J_{m}$. These zeros can be approximated by $j_{m n}^{\prime} \simeq$ $\left(n+\frac{1}{2} m-\frac{3}{4}\right) \pi$. Consequently, the difference $\Delta k=\left|\hat{k}_{j+1}-\hat{k}_{j}\right|$ between two adjacent axial wavenumbers that lie away from the real axis is approximately constant $\pi / \sqrt{1-M^{2}}$, whereas for modes that lie close to the real axis $\Delta k$ depends on $\omega$. This motivates the choice

$$
\epsilon_{t o l}=\tilde{\epsilon}_{t o l} \Delta k_{r e f}
$$

where $\Delta k_{r e f}:=\left|\hat{k}_{j+1}-\hat{k}_{j}\right|$ depends on the mode considered and $\tilde{\epsilon}_{t o l}$ is a parameter, equal for all modes, that has to be chosen. We also add an upper limit $h_{\max }$ to the step size. We finally have:

$$
h_{j+1}=\min \left[h_{j} \sqrt{\frac{\Delta k_{r e f} \tilde{\epsilon}_{t o l}}{\epsilon_{j}}}, h_{\text {max }}\right]
$$

In some cases, a small change in $\lambda$ results in a big change in the solution. It is even possible to 'jump' to another mode. Therefore, we restart from the former solution with a halved step size when the error is too large, i.e, when $\epsilon>\epsilon_{\max }$.

There is some trade-off in choosing the parameters $\tilde{\epsilon}_{t o l}, \tilde{\epsilon}_{\max }, h_{\max }$ and the initial step size $h_{1}$; we would like to travel through the path quickly, while at the same time maintaining a certain confidence that we do not 'jump' to another mode.

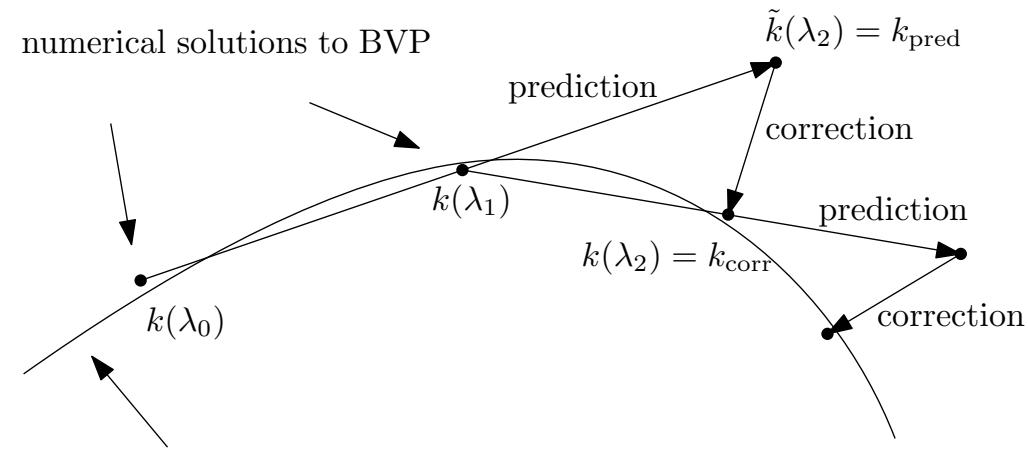

curve that satisfies BVP

$$
\epsilon=\left|k_{\text {pred }}-k_{\text {corr }}\right|
$$

Figure 3. Prediction-correction scheme. 


\section{Numerical results}

\section{VII.A. Exponentially decaying eigenfunctions}

Our numerical solution has obviously been thoroughly tested in the cases of a uniform mean flow and mean temperature, where analytic solutions are available to compare with.

For non-trivial cases of non-uniform mean flow it is much harder to find suitable test cases. A possible configuration is a strongly non-uniform (parabolic) mean flow with upstream running modes of sufficiently high frequency. The modes will refract to the part of the medium with the lowest (effective) sound speed [27], which is in this case the duct center. The wave becomes exponentially small near the wall, which may be challenging for numerical methods like shooting. However, the WKB solution of section (III.A) is very applicable and will therefore be used here for making a comparison.

Consider the case where $\omega=25, m=5, Z=2-\mathrm{i}$, the mean flow temperature is unity, and the mean flow velocity is $M(r)=\frac{2}{3}\left(1-\frac{1}{2} r^{2}\right)$. The first 6 upstream running eigenfunctions, decaying exponentially towards the wall, are depicted in Figure 4 . A comparison of the axial wavenumbers found by COLNEW with the wavenumbers determined by the quantization condition (12) shows an excellent agreement, see Table 1 . As there is little or no influence of the impedance wall, the wave numbers are practically real. Only for the higher order modes the damping of the wall becomes little by little effective as the imaginary parts of the wave numbers become negative (note: left-running modes with $\exp (-\mathrm{i} \omega t)$ convention).
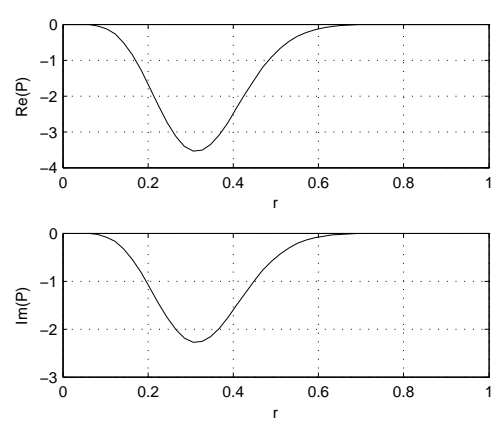

(a) $n=1$
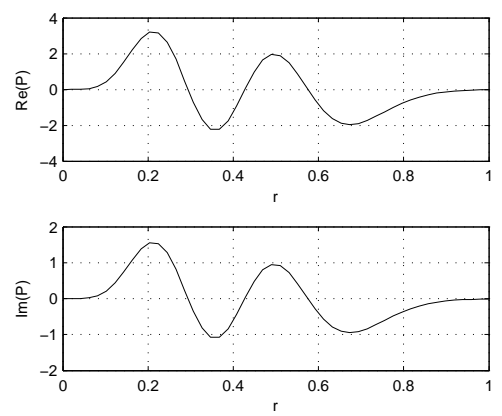

(d) $n=4$
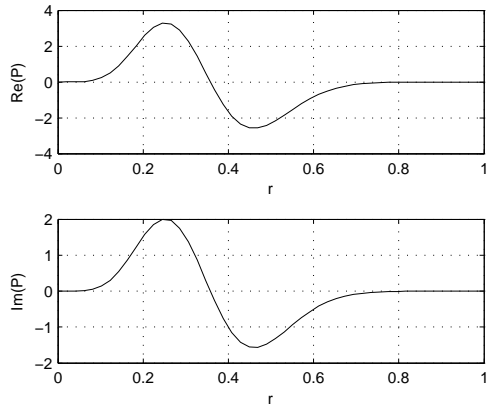

(b) $n=2$
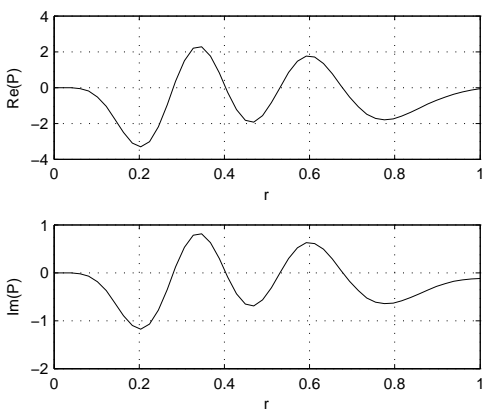

(e) $n=5$
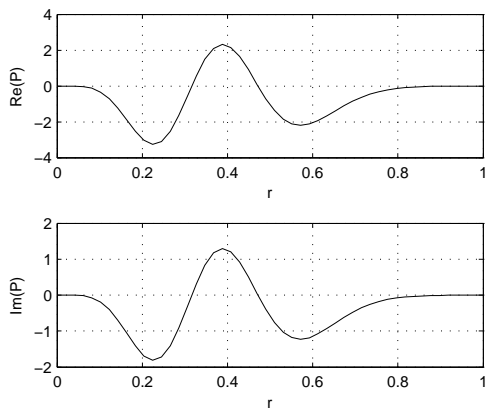

(c) $n=3$
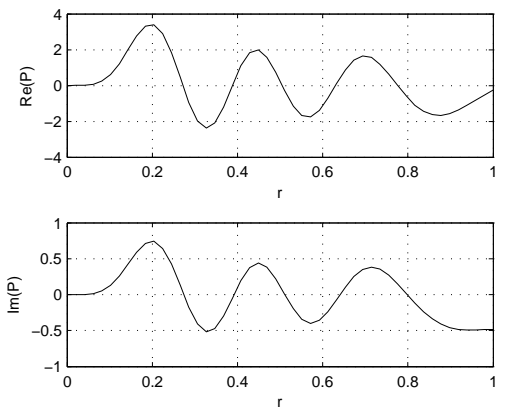

(f) $n=6$

Figure 4. Upstream-running modes in non-uniform mean flow, refracting away from the duct wall.

\begin{tabular}{lll}
\hline$n$ & $k$ according to radial WKB & $k$ found by COLNEW \\
\hline 1 & -60.470050 & -60.4392 \\
2 & -55.761477 & -55.7281 \\
3 & -51.134220 & $-51.0980-0.0000 \mathrm{i}$ \\
4 & -46.605341 & $-46.5659-0.0003 \mathrm{i}$ \\
5 & -42.195806 & $-42.1422-0.0212 \mathrm{i}$ \\
6 & -37.931062 & $-37.5622-0.3254 \mathrm{i}$ \\
\hline
\end{tabular}

Table 1. Modal wave numbers $k$, corresponding to figures (4), found from WKB's quantization condition and by COLNEW. 


\section{VII.B. APU exhaust duct with slowly varying impedance}

In this section we show some numerical results for test cases motivated by a realistic APU exhaust duct, having a length of $1 \mathrm{~m}$ and a radius of $0.15 \mathrm{~m}$. We choose the reference temperature $T_{0}=700 \mathrm{~K}$ and the mean density $\rho_{0}=0.5 \mathrm{kgm}^{-3}$, corresponding to a reference sound speed of $c_{0}=549 \mathrm{~ms}^{-1}$. We consider cases where $\operatorname{Im} Z(x)$ varies linearly with fixed $\operatorname{Re} Z=1.5$, and cases where the liner is modeled as a Helmholtz resonator [28]. To assess the applicability of the WKB method and estimate the truncation error in the WKB expansion (which is $O(\varepsilon)$, see (19)), we need to estimate $\varepsilon$. This can be done by noting that if $Z$ varies typically over a length scale $L$, we have

$$
\frac{R}{Z} \frac{\mathrm{d}}{\mathrm{d} x} Z(x / L)=\frac{R}{L} \frac{Z^{\prime}(x / L)}{Z}=O(\varepsilon),
$$

since by assumption $Z^{\prime} / Z=O(1)$. We plot contour lines of the pressure field, with one $n$-th order radial mode as input at $x=0$ (usually, $n=1$ ). For cases of uniform mean flow velocity and temperature we compare the (axial) WKB results with the BAHAMAS results. The eigenfunctions are normalized according to

$$
\int_{0}^{1}|\psi|^{2} r \mathrm{~d} r=1, \quad \psi^{\prime}(1) \in \mathbb{R}^{+},
$$

which is the convention used in BAHAMAS.

For the WKB results we first compute the eigenfunctions and axial wavenumbers for $200 x$-values in the range from 0 to 1 by using our mode solver. (Note that the small parameter $\varepsilon$ only acts as a bookkeeping parameter since we can write everything as a function of slow variable $X$.) Subsequently, the amplitudes $N(X)$ are computed using (29) or using the special cases (32) and (31). The integrals over $\eta$ are computed by using a trapezoidal rule, which enables us to use all computed eigenfunctions (for all $X$-values) for the field plot. We use a higher number of $X$-values than necessary to achieve the required accuracy in order to have smooth contour plots. The integrals over $r$ in (28b), (30) and (32) are computed by using the QUADPACK [29] code (which is based on an adaptive Gauss-Kronrod rule), the accuracy of which actually commensurates with the other integral rule. We motivate this choice by noting that the eigenfunction (and hence the integrand in the radial direction) can be oscillatory, while the integrands in the axial direction are slowly varying. For non-uniform mean flow we need to compute $f(X)$. Working out (28b) yields

$$
\begin{aligned}
f(X)=\mathrm{i} \int_{0}^{1}\left\{-\frac{2 \omega \psi \bar{T}}{\Lambda^{5}} \frac{\mathrm{d} \bar{u}}{\mathrm{~d} r}\left[\Lambda \frac{\partial^{2} \psi}{\partial r \partial X}+\bar{u} \frac{\mathrm{d} \mu}{\mathrm{d} X} \frac{\partial \psi}{\partial r}\right]\right. & \left.+\frac{\psi}{\Lambda^{2}}\left[\left(\bar{T}-\bar{u}^{2}\right) \frac{\mathrm{d} \mu}{\mathrm{d} X} \psi+2(\bar{u} \Lambda+\mu \bar{T}) \frac{\partial \psi}{\partial X}\right]\right\} r \mathrm{~d} r \\
& -\frac{\bar{u} \psi}{\omega \Lambda^{2} Z^{2}}\left[2 \Lambda Z \frac{\partial \psi}{\partial X}-\left(Z \bar{u} \frac{\mathrm{d} \mu}{\mathrm{d} X}+2 \Lambda \frac{\mathrm{d} Z}{\mathrm{~d} X}\right) \psi\right]_{r=1}
\end{aligned}
$$

The $X$ derivatives (which are not available analytically) are computed by using second order finite differences.

To test the WKB approach we first let $Z(x)$ vary linearly from $1.5-\mathrm{i}$ to $1.5+\mathrm{i}$, which corresponds to an estimated $\varepsilon=0.2$. Figure 5 shows the piecewise impedance for the segments that are used in BAHAMAS. From Figure 6(a) and Figure 6(b) it is clear that the difference between constant impedance and axially varying impedance is significant, so the $x$-dependency of $Z$ has to be taken into account. Figure 6(b) shows that the WKB and the BAHAMAS results agree reasonably well for this not very small choice of $\varepsilon$. From Figure 6(c)), with the same parameter values and mean flow mass flux as before but a parabolic mean flow profile, we conclude that the effect of a non-uniform mean flow is not to be neglected. The present difference is explained by the fact that downstream running sound waves are refracted towards the lined wall [27], resulting in more damping.

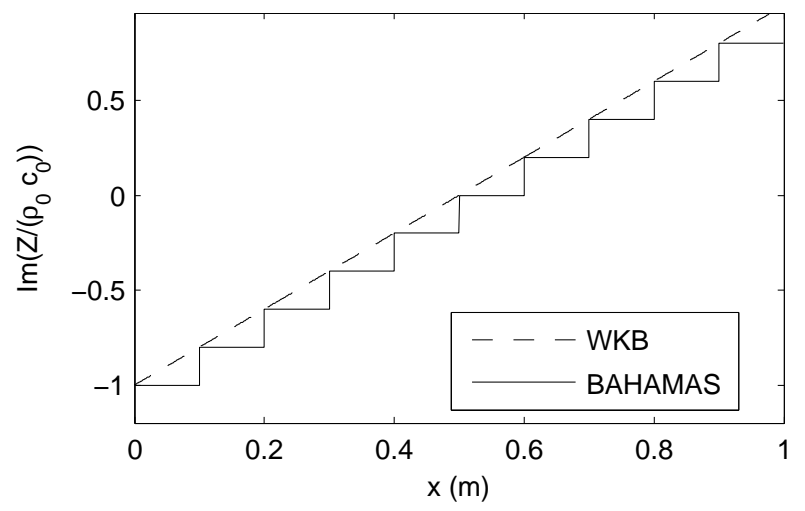

Figure 5. $Z$ varies linearly from $1.5-i$ to $1.5+i, \varepsilon \approx 0.2$. 
In the next configuration, we let $Z(x)$ vary linearly from $1.5-2 \mathrm{i}$ to $1.5+2 \mathrm{i}$ along the same interval, which corresponds to a higher estimated $\varepsilon$ of 0.4 . The truncation error in the WKB approximation is now larger and we may expect a bigger difference between the WKB and the BAHAMAS solutions. This is indeed the case, as is shown in Figure 7. Near $x=0.7 \mathrm{~m}$, some signs of intermodal scattering are visible, which is explicitly not taken into account by the WKB method.

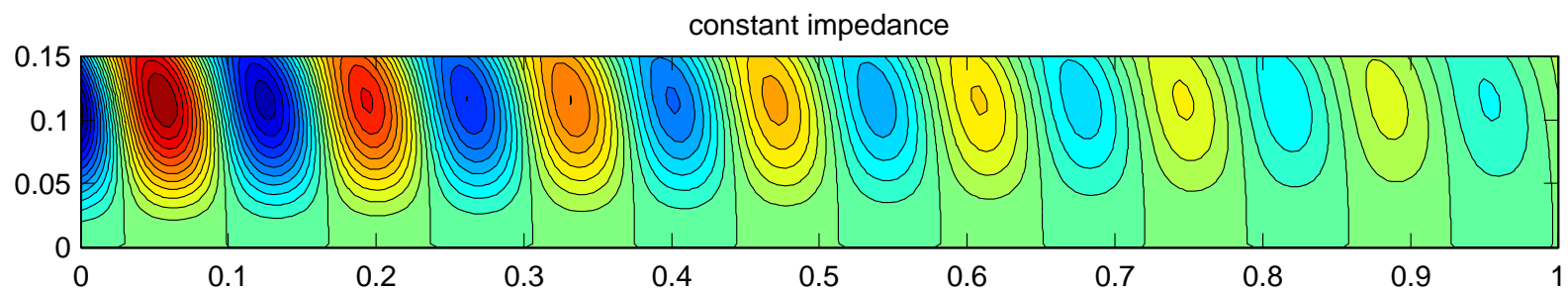

(a) $Z=1.5-\mathrm{i}$, uniform mean flow velocity $M_{0}=0.3$.
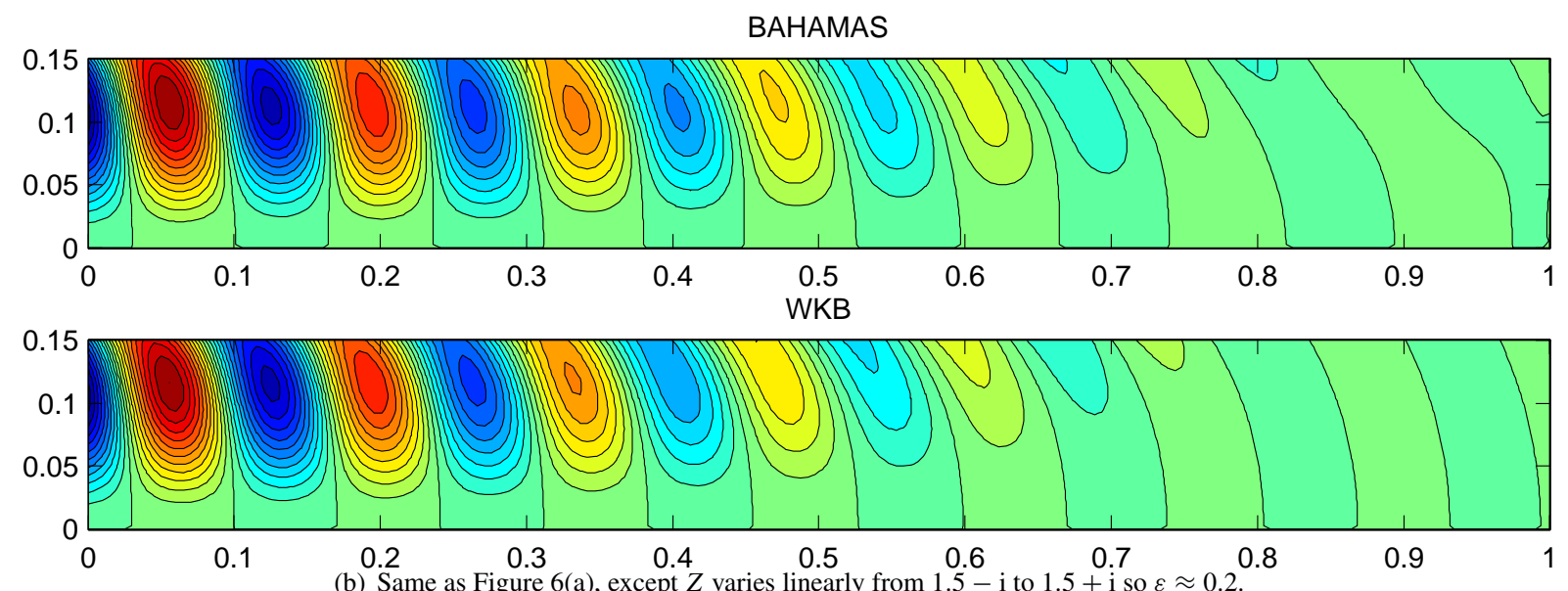

WKB

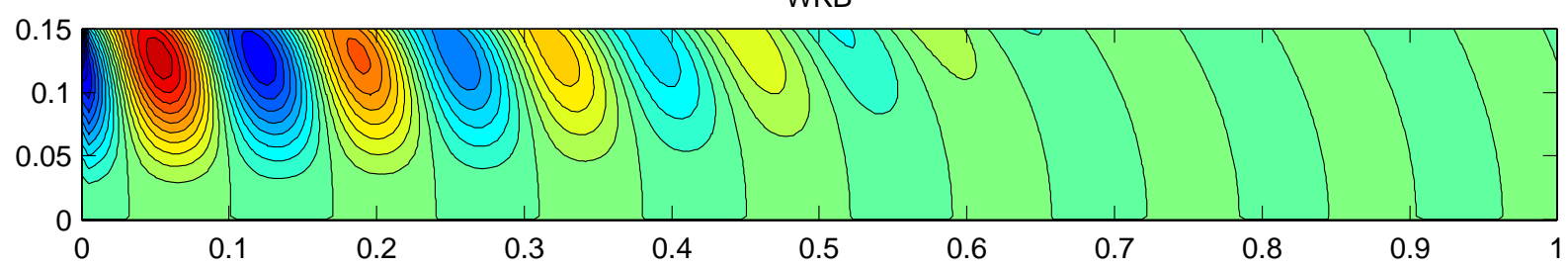

(c) Same as Figure 6(b) except mean flow velocity $M(r)=M_{0} \frac{4}{3}\left(1-\frac{1}{2} r^{2}\right)$ with $M_{0}=0.3$.

Figure 6. $\omega=10, m=2, n=1$
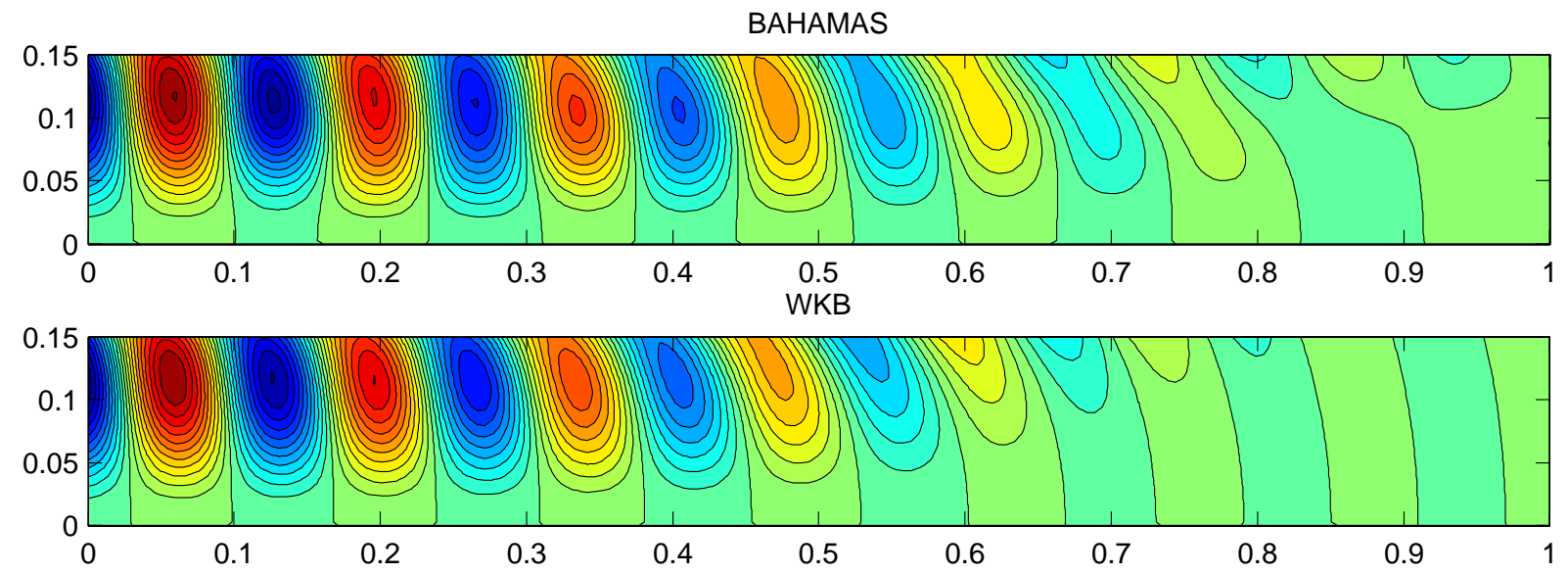

Figure 7. $\omega=10, m=2, n=1$, uniform mean flow velocity $M_{0}=0.3, Z$ varies linearly from $1.5-2 \mathrm{i}$ to $1.5+2 \mathrm{i}$ so $\varepsilon \approx 0.4$.

To evaluate the applicability of the WKB method for a realistic APU exhaust duct geometry, we now consider a locally reacting liner with a cell depth $d(x)$ that is axially varying from $7 \mathrm{~cm}$ to $1 \mathrm{~cm}$ along the duct. The 
dimensional impedance is modeled as a Helmholtz resonator, described (with $\mathrm{e}^{-\mathrm{i} \omega t}$ convention) as

$$
\hat{Z}=\hat{Z}_{0}+\mathrm{i} \rho_{0} c_{0} \cot \left(\hat{\omega} d / c_{0}\right)
$$

where $\hat{\omega}$ is the dimensional frequency and $\hat{Z}_{0}$ is the face sheet impedance

$$
\hat{Z}_{0}=\hat{R}_{0}-\mathrm{i} \hat{\omega} \tilde{m} \text {. }
$$

We choose a facing sheet resistance of $\hat{R}_{0}=400 \mathrm{kgm}^{-2} s^{-1}$, and a mass reactance of $\tilde{m}=0.001 \mathrm{kgm}^{-2}$. We remark that this is only a model and the reference sound speed $c_{0}$ may be different from the sound speed at the wall for non-uniform temperature profiles. Figure 8 shows the imaginary part of the impedance as a function of $x$ for two different frequencies. Note that for $\omega=10$ resonance occurs; close to $x=0.4 \mathrm{~m}$ the liner behaves as a hard wall.
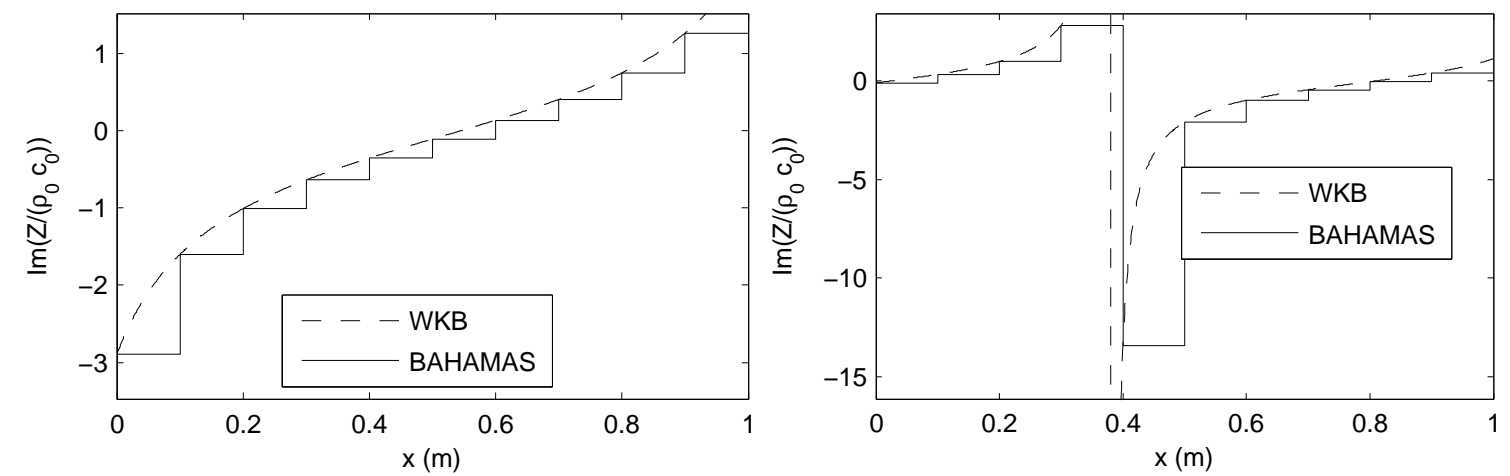

Figure 8. $Z$ modeled as Helmholtz resonator with liner depth that varies linearly from $7 \mathbf{c m}$ to $1 \mathrm{~cm}$. Left: $\omega=6(\varepsilon \approx 0.3)$, right: $\omega=10($ resonance occurs).

Figure 9 show the acoustic field for $\omega=6$. On the interval considered, there is no location where the Helmholtz resonator is in resonance; $Z(x)$ is slowly varying with an estimated $\varepsilon=0.3$. The WKB and BAHAMAS results show rather good agreement, about what can be expected from this value of $\varepsilon$.
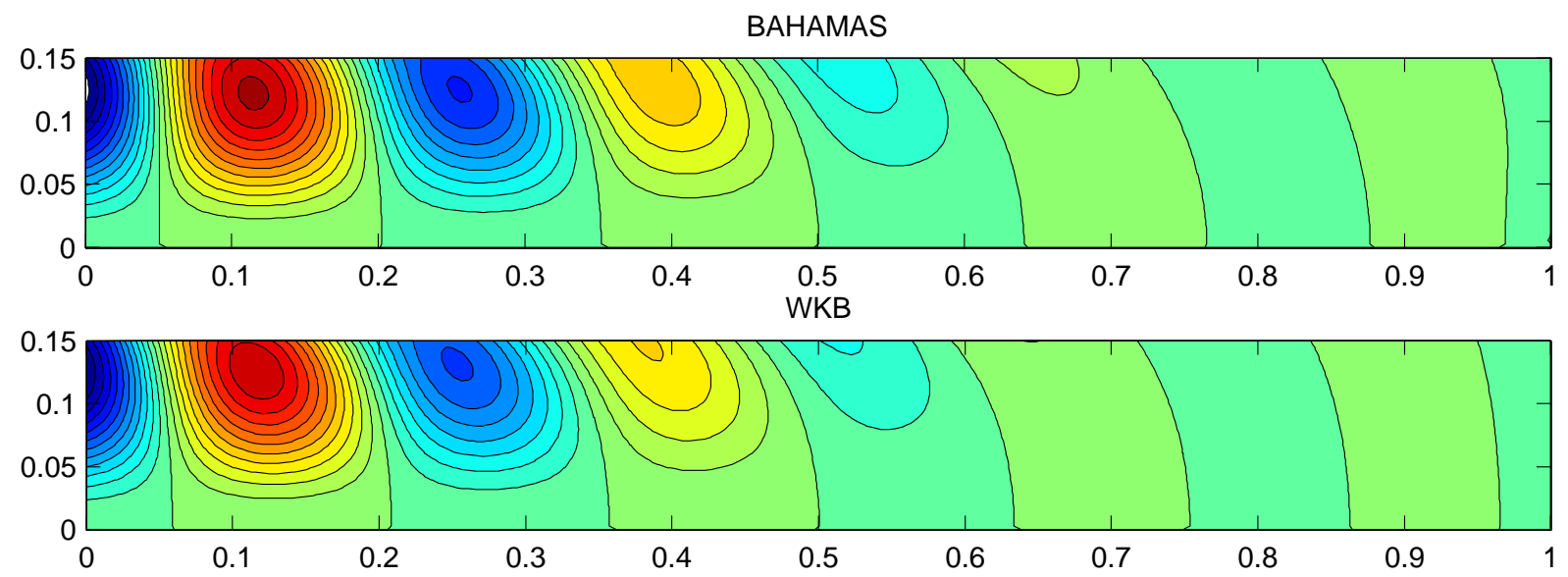

Figure 9. $\omega=6, m=2, n=1$, uniform mean flow velocity $M_{0}=0.3, Z$ modeled as Helmholtz resonator with liner depth that varies linearly from $7 \mathrm{~cm}$ to $1 \mathrm{~cm}$.

However, when $\omega=10$ we do have resonance near $x=0.4$ so the assumption of a slowly varying $Z(x)$ is, at least near this point, not valid anymore. Indeed, this can be identified from Figure 10, where the region of resonance seems to excite the second radial mode, an effect which cannot be described by (straight-forward) application of the WKB method.

With a realistic APU exhaust duct, cool air is let in near $x=0$ along the wall (see Figure 1 ). This produces a strong radial temperature gradient. We modeled this by a tanh-type profile, given in Figure 11. The effect of this temperature gradient is that it creates effectively two concentric ducts, each with its own propagation properties. These duct fields are not completely independent of each other. Sound waves from the center region (with the highest sound speed) will refract (by a form of Snell's law) to the colder annular region. However, sound in the annular region refract only if the angle between duct axis and their propagation direction is not too small. Otherwise, the annular region will act as a duct on its own.

This is illustrated in Figure 12, where the fields are plotted for the first two right-running radial modes. In case of the first mode, the field is virtually only existent in the colder outer region. The field of the second mode exists in both, but such that the sound waves refract from inside to outside. 

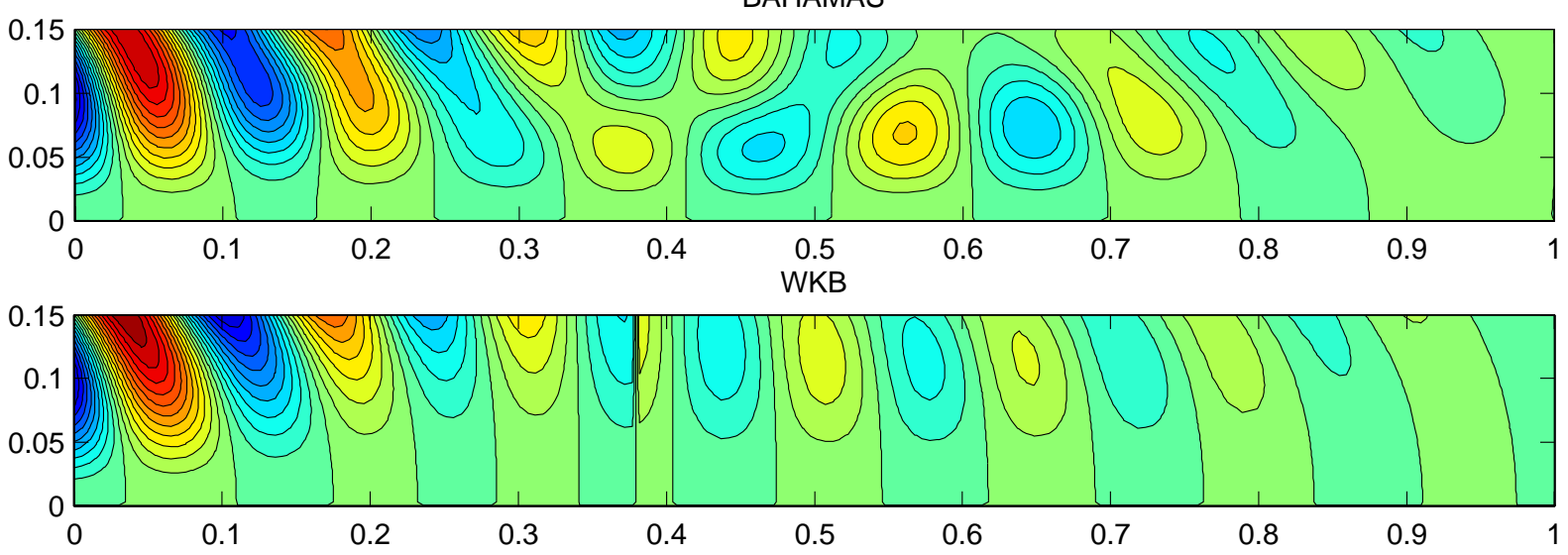

Figure 10. $\omega=10, m=2, n=1$, uniform mean flow velocity $M_{0}=0.3, Z$ modeled as Helmholtz resonator with liner depth that varies linearly from 7 cm to $1 \mathrm{~cm}$.

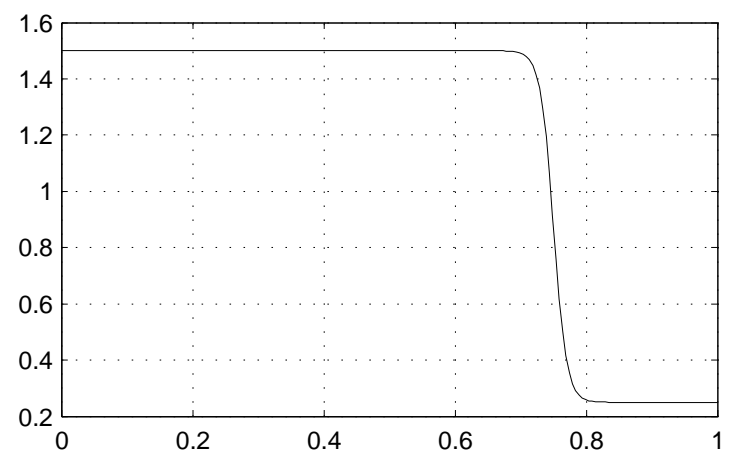

Figure 11. Temperature profile $T(r)=\frac{1}{4}+\frac{5}{8}\left[1+\tanh \left(50\left(\frac{3}{4}-r\right)\right)\right]$.

\section{Conclusions}

The WKB method for slowly varying modes was applied to a typical geometry of an APU exhaust.

The numerical evaluation of the mode shape functions (solutions of the Pridmore-Brown equation) is realized by application of the freely available, standard implementation COLNEW, a code based on a general collocation method for non-linear boundary value problems. In order to find all modes, the modal solver is embedded in a path-following (or continuation) procedure with automatic adaptation of the step size.

In order to test the numerical Pridmore-Brown solutions for non-trivial situations, favourable comparisons have been made with analytic approximate solutions of WKB type (in radial direction, not to be confused with the axial WKB solutions of modes varying slowly along the duct).

To demonstrate the value of our slowly varying modal solution, a number of tests have been made. For a wall impedance that varies slowly in axial direction - slow enough to be within the range of applicability of the WKB method - the results agree well (depending on the small parameter) with the BAHAMAS reference solution based on uniform flow and a piecewise constant impedance. On the other hand, the WKB solution lacks to capture typical phenomena associated with resonance that invariably occurs when the impedance wall is of Helmholtz resonator type. This is not unexpected as the liner reactance changes sign via "infinity" along a short interval and therefore is locally not slowly varying. In reality we will observe intermodal scattering, which is, by assumption, excluded in a WKB solution.

Finally, a few typical results are presented for non-uniform flow and a strong temperature gradient.

\section{Acknowledgments}

We appreciated gratefully the fruitful discussions with L.P.J. Kamp.

\section{References}

${ }^{1}$ Th. Nodé-Langlois, P. Sijtsma, S. Moal, and F. Vieuille, "Modelling of Non-Locally Reacting Acoustic Treatments for Aircraft Ramp Noise Reduction,” No. AIAA 2010-3769, June 7-9 2010.

${ }^{2}$ D.C. Pridmore-Brown, "Sound propagation in a fluid flowing through an attenuating duct," J. Fluid Mech., Vol. 4, 1958, pp. $393-406$. 

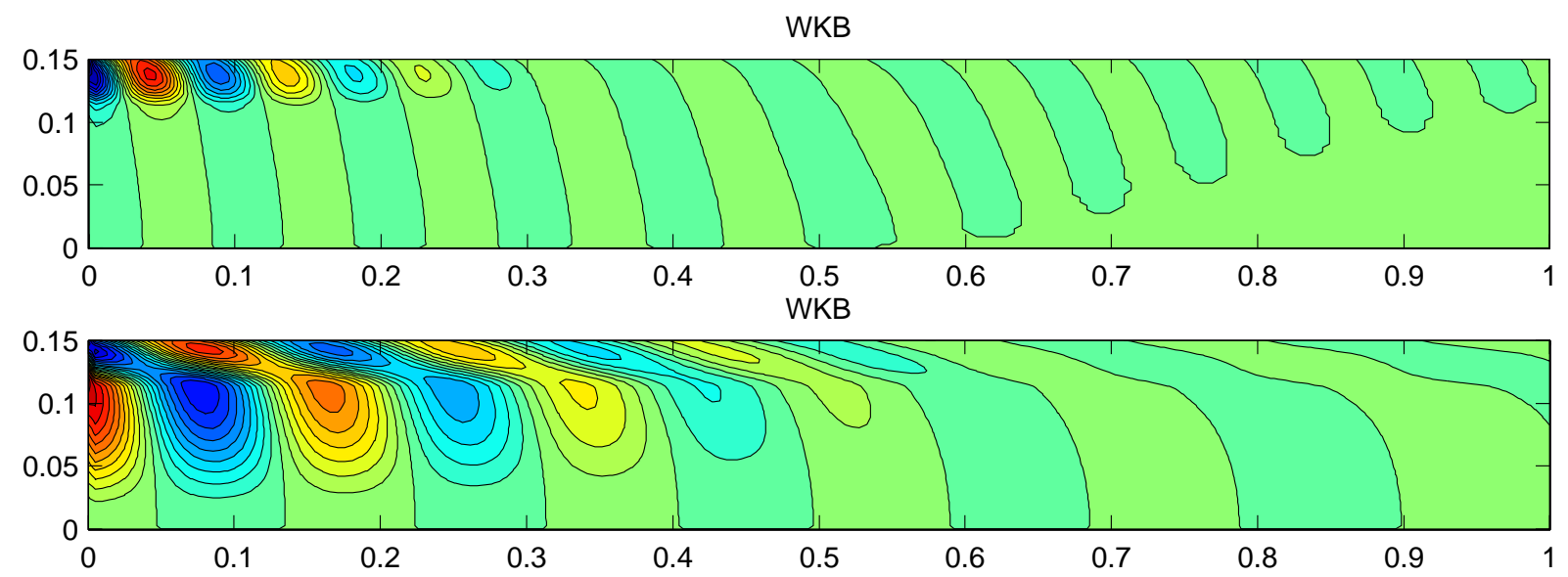

Figure 12. $\omega=10, m=2, n=1$ and $n=2$, uniform mean flow velocity $M_{0}=0.3$, strong temperature gradient (see Figure 11], $Z(x)$ varies linearly from $1.5-\mathrm{i}$ to $1.5+\mathrm{i}$.

${ }^{3}$ G.G. Vilenski and S.W. Rienstra, "On hydrodynamic and acoustic modes in a ducted shear flow with wall lining," Journal of Fluid Mechanics, Vol. 583, 2007, pp. 45-70.

${ }^{4}$ A.H. Nayfeh, Introduction to Perturbation Techniques, John Wiley and Sons, Inc., 1993.

${ }^{5}$ S.W. Rienstra, "Sound propagation in slowly varying lined flow ducts of arbitrary cross-section," Journal of Fluid Mechanics, Vol. 495, 2003, pp. 157-173

${ }^{6} \mathrm{~W}$. Lazeroms, Sound propagation in slowly varying lined ducts with temperature gradients, Master's thesis, Eindhoven University of Technology, December 2010.

${ }^{7}$ A.J. Cooper and N. Peake, "Propagation of unsteady disturbances in a slowly varying duct with mean swirling flow," J. Fluid Mech., Vol. 445, 2001, pp. 207-234.

${ }^{8}$ N. Peake and A.J. Cooper, "Acoustic propagation in ducts with slowly varying elliptic cross-section," J. Sound Vib., Vol. 243, 2001, pp. 381-401.

${ }^{9}$ A.J. Cooper, "Effect of mean entropy on unsteady disturbance propagation in a slowly varying duct with mean swirling flow," Journal of Sound and Vibration, Vol. 291, 2006, pp. 779-801.

${ }^{10}$ E.J. Brambley and N. Peake, "Propagation of unsteady disturbances in a slowly varying duct with mean swirling flow," J. Fluid Mech., Vol. 596, 2008, pp. 387-412.

${ }^{11}$ S.W. Rienstra, "Sound transmission in slowly varying circular and annular ducts with flow," Journal of Fluid Mechanics, Vol. 380, 1999, pp. 279-296.

${ }^{12}$ S.W. Rienstra, "A numerical comparison between multiple-scales and finite-element solution for sound propagation in lined flow ducts," Journal of Fluid Mechanics, Vol. 437, 2001, pp. 367-384.

${ }^{13} \mathrm{G}$. Bader and U. Ascher, "A New Basis Implementation for a Mixed Order Boundary Value ODE Solver," SIAM J. Sci. and Stat. Comput., Vol. 8, No. 4, July 1987, pp. 483-500.

${ }^{14}$ W. Eversman, "Computation of axial and transverse wave numbers for uniform two-dimensional ducts with flow using a numerical integration scheme," Journal of Sound Vibration, Vol. 41, jul 1975, pp. 252-255.

${ }^{15}$ S.W. Rienstra, "A classification of duct modes based on surface waves," Wave Motion, Vol. 37, No. 2, 2003, pp. 119-135.

${ }^{16}$ E.L. Allgower and K. Georg, Introduction to Numerical Continuation Methods, SIAM, 2003.

${ }^{17}$ K.U. Ingard, "Influence of fluid motion past a plane boundary on sound reflection, absorption, and transmission," J. Acoust. Soc. Am., Vol. 31, 1959, pp. 1035-1036.

${ }^{18}$ M.K. Myers, "On the acoustic boundary condition in the presence of flow," J. Sound Vib., Vol. 71, 1980, pp. 429-434.

${ }^{19}$ S.W. Rienstra and M. Darau, "Boundary Layer Thickness Effects of the Hydrodynamic Instability along an Impedance Wall," J. Fluid Mech., Vol. 671, 2011, pp. 559-573.

${ }^{20} \mathrm{~N}$. Fröman and P.O. Fröman, JWKB approximation: contributions to the theory, North-Holland, Amsterdam, 1965.

${ }^{21}$ P. Sijtsma and H. van der Wal, "Modelling a Spiralling Type of Non-Locally Reacting Liner," 9th AIAA/CEAS Aeroacoustics Conference and Exhibit, Hilton Head, South Carolina, No. AIAA-2003-3308, 2003.

${ }^{22}$ G.G. Vilenski and S.W. Rienstra, "Numerical study of acoustic modes in ducted shear flow," Journal of Sound and Vibration, Vol. 307, 2007, pp. 610-626.

${ }^{23}$ G. Boyer, Estelle Piot, and Jean-Philippe Brazier, "Theoretical investigation of hydrodynamic surface mode in a lined duct with sheared flow and comparison with experiment," Journal of Sound and Vibration, Vol. 330, 2011, pp. 1793-1809. 1282.

${ }^{24}$ E.J. Brambley, "A well-posed boundary condition for acoustic liners in straight ducts with flow," AIAA Journal, Vol. 49, 2011, pp. 1272-

${ }^{25}$ U. Ascher, J. Christiansen, and R.D. Russel, "Collocation software for Boundary-Value ODEs," ACM Transactions on Mathematical Software, Vol. 7, No. 2, June 1981, pp. 209-222.

${ }^{26}$ http://www.netlib.org/ode/colnew.f.

${ }^{27}$ S.W. Rienstra and A. Hirschberg, "An Introduction to Acoustics," Tech. Rep. IWDE 92-06, Technische Universiteit Eindhoven, 19922004-2011, http://www.win.tue.nl/ sjoerdr/papers/boek.pdf.

${ }^{28}$ S.W. Rienstra, "Impedance Models in Time Domain including the Extended Helmholtz Resonator Model," 12th AIAA/CEAS Aeroacoustics Conference, No. AIAA 2006-2686, Cambridge MA, 2006.

${ }^{29}$ R. Piessens, E. De Doncker-Kapenga, and C.W. Überhuber, QUADPACK: a subroutine package for automatic integration, Springer, 1983, http://www.netlib.org/quadpack/. 